\title{
RECURSIVE EQUIVALENCE TYPES OF VECTOR SPACES
}

\author{
ALAN G. HAMILTON \\ (Received 14 August 1972; revised 19 February 1973) \\ Communicated by J. N. Crossley
}

We consider subspaces of a vector space $U_{F}$, which is countably infinite dimensional over a recursively enumerable field $F$ with recursive operations, where the operations in $U_{F}$ are also recursive, and where, of course, $F$ and $U_{F}$ are sets of natural numbers. It is the object of this paper to investigate recursive equivalence types of such vector spaces and the ways in which their properties are analogous to and depend on properties of recursive equivalence types of sets.

\section{Introduction}

The reader is referred to Dekker [2] for details of the construction of $U_{F}$. For the sake of convenience we shall write addition and scalar multiplication of vectors in $U_{F}$ in the usual way, namely $v_{1}+v_{2}$ and $x v_{1}$ (for $x \in F$ ), although of course these operations are not the same as addition and multiplication of natural numbers. There will be no confusion. Let $N$ denote the set of natural numbers. If $V$ and $W$ are subspaces of $U_{F}$ and $V$ is a subspace of $W$, we write $V \subseteq W$. It will be useful to pick out one recursively enumerable basis of $U_{F}$. We shall call it the standard basis and denote it $\left\{p_{i}: i<\omega\right\}$.

The following is a brief exposition of the results from Dekker [2] and Hamilton [4] that we shall need. The reader is assumed to be familiar with recursive equivalence types, as in, say, Dekker and Myhill [3].

Definition 1.1. If $V \subseteq U_{F}$ and $A$ is a basis of $V$, then $A$ is an $\alpha$-basis of $V$ if $A$ is contained in a r.e. linearly independent subset of $U_{F}$.

Let $D$ be the set of all subspaces of $U_{F}$ which have an $\alpha$-basis.

Lemma 1.2. (i) An r.e. basis is an $\alpha$-basis.

(ii) If $A$ is an $\alpha$-basis of $V$ then there is a uniform effective procedure for obtaining, given $v \in V$, the (finite) linear combination of vectors of $A$ which is equal to $v$.

If $A$ is a subset of $U_{F}$, then the subspace generated by $A$ will be denoted by $V(A)$. One of the principal results of [4] is: 
THEOREM 1.3. If $V \subseteq U_{F}$ and $A$ and $B$ are two $\alpha$-bases of $V$, then $A$ and $B$ are recursively equivalent.

This enables us to make the following definition.

Definition 1.4. If $V \in D$, then the $\alpha$-dimension of $V$ (written $\operatorname{dim}_{\alpha} V$ ) is $\operatorname{RET}(A)$ where $A$ is any $\alpha$-basis of $V$.

DEFINITION 1.5. If $V$ and $W$ are subspaces of $U_{F}$ (not necessarily members of $D$ ), then $V$ is $\alpha$-isomorphic to $W$ (written $V \simeq W$ ) if there is a one-one partial recursive function $p$ such that

(i) $\delta p$ and $\rho p$ are subspaces of $U_{F}$. ( $\delta p$ and $\rho p$ are the domain and range of $p$ ),

(ii) $V \subseteq \delta p$,

(iii) $p(V)=W$,

(iv) $p$ is a classical isomorphism between $\delta p$ and $\rho p$.

It is easy to show that $\simeq$ is an equivalence relation. We call the equivalence classes V-R.E.T.S.

THEOREM 1.6. If $V, W \in D$, then $V \simeq W$ if and only if $\operatorname{dim}_{\alpha} V=\operatorname{dim}_{\alpha} W$. Let $\Omega_{V}=\{V-R E T(V): V \in D\}$.

Definition 1.7. If $T \in \Omega_{V}$, then the $\alpha$-dimension of $T$ (written $\operatorname{dim}_{\alpha} T$ ) is $\operatorname{dim}_{x} V$, where $V$ is any element of $T$.

Let $\Lambda_{V}=\left\{T \in \Omega_{V}: \operatorname{dim}_{\alpha} T \in \Lambda\right\}$.

From Theorem 1.6 we immediately deduce:

COROLLARY 1.8. If $T_{1}, T_{2} \in \Omega_{V}$, then $T_{1}=T_{2}$ if and only if $\operatorname{dim}_{\alpha} T_{1}=\operatorname{dim}_{\alpha} T_{2}$.

Finally, it is not difficult to show that for any R.E.T. $X$ there is an element $T$ of $\Omega_{V}$ such that $\operatorname{dim}_{\alpha} T=X$.

\section{Operations}

We define addition of subspaces of $U_{F}$ analogously to the separable sum of subsets of $N$, in order that the sum may be well-defined on space-types.

Define functions $q_{1}, q_{2}: U_{F} \rightarrow U_{F}$ as follows: If $v=\left(v_{0}, \cdots, v_{k}\right)$ relative to the standard r.e. basis $\left\{p_{i}\right\}$, let

and

$$
q_{1}(v)=\left(v_{0}, 0, v_{1}, 0, \cdots, v_{k}\right),
$$

$$
q_{2}(v)=\left(0, v_{0}, 0, v_{1}, \cdots, 0, v_{k}\right) .
$$

Now if $V$ and $W$ are subspaces of $U_{F}$ then $q_{1}(V) \cap q_{2}(W)=\{0\}$, and the sets $q_{1}(V)-\{0\}$ and $q_{2}(W)-\{0\}$ are recursively separable.

Definition 2.1. (i) If $V$ and $W$ are subspaces of $U_{F}$, let 


$$
V+W=\left\{v+w: v \in q_{1}(V) \& w \in q_{2}(W)\right\} .
$$

(ii) If $V \subseteq U_{F}$, let $V .1=V$, and for $n>1$, let $V \cdot n=V \cdot(n-1)+V$.

Note that this addition is neither commutative nor associative, but we are principally interested in the addition induced on V-R.E.T.S, which is both.

LEMMA 2.2. If $V_{1} \simeq V_{2}$ and $W_{1} \simeq W_{2}$, then $V_{1}+W_{1} \simeq V_{2}+W_{2}$.

Proof. Suppose that $p: V_{1} \simeq V_{2}$ and $q: W_{1} \simeq W_{2}$. Then let

$$
p^{\prime}=q_{1} \circ p \circ q_{1}^{-1}: q_{1}\left(V_{1}\right) \simeq q_{1}\left(V_{2}\right)
$$

and

$$
q^{\prime}=q_{2} \circ q \circ q_{2}^{-1}: q_{2}\left(W_{1}\right) \simeq q_{2}\left(W_{2}\right) .
$$

Given $u \in V_{1}+W_{1}$, i.e. $u=v+w$ for some $v \in V_{1}$ and $w \in W_{1}$, this expression is unique and we can effectively obtain $v$ and $w$. Let

$$
r(u)=p^{\prime}(v)+q^{\prime}(w)
$$

for such $u$.

It is not difficult to verify that $r$ is an $\alpha$-isomorphism between $V_{1}+W_{1}$ and $V_{2}+W_{2}$.

CoRollary 2.3. + and $n$ for each $n \in N$ induce well defined operations on V-R.E.T.S.

Definition 2.4. If $T_{1}$ and $T_{2}$ are V-R.E.T.s, then $T_{1}+T_{2}$ is defined to be $V-R E T\left(V_{1}+V_{2}\right)$, where $V_{1} \in T_{1}$ and $V_{2} \in T_{2}$. As before, also, if $T$ is a $V-R . E . T$, define $T .1=T$ and, for $n>1$, T.n $=T .(n-1)+T$.

THEOREM 2.5. Addition of V-R.E.T.s is commutative and associative.

THEOREM 2.6. If $V_{1}, V_{2} \in D$ and $V=V_{1}+V_{2}$, then $V \in D$ and $\operatorname{dim}_{\alpha} V=$ $\operatorname{dim}_{\alpha} V_{1}+\operatorname{dim}_{\alpha} V_{2}$.

Proof. Suppose that $A_{1}$ and $A_{2}$ are $\alpha$-bases of $V_{1}$ and $V_{2}$ respectively. Then $q_{1}\left(A_{1}\right)$ and $q_{2}\left(A_{2}\right)$ are recursively separable, and $q_{1}\left(A_{1}\right) \cup q_{2}\left(A_{2}\right)$ is an $\alpha$-basis of $V$. Therefore $V \in D$. Now

$$
\begin{aligned}
\operatorname{dim}_{\alpha} V & =\operatorname{RET}\left(q_{1}\left(A_{1}\right) \cup q_{2}\left(A_{2}\right)\right) \\
& =\operatorname{RET}\left(q_{1}\left(A_{1}\right)\right)+\operatorname{RET}\left(q_{2}\left(A_{2}\right)\right) \\
& =\operatorname{dim}_{\alpha} V_{1}+\operatorname{dim}_{\alpha} V_{2} .
\end{aligned}
$$

Corollary 2.7. If $T_{i} \in \Omega_{V}$ and $n_{i} \in N$ for $1 \leqq i \leqq k$, and $T=\sum_{i=1}^{k} T_{i} \cdot n_{i}$, then $T \in \Omega_{V}$ and $\operatorname{dim}_{\alpha} T=\sum_{i=1}^{k}\left(\operatorname{dim}_{\alpha} T_{i}\right) . n_{i}$.

Now we consider the order relation $\leqq$ on spaces and space types. Note that our use of the symbol $\leqq$ here is different from Dekker's use in [2]. 
DeFINITION 2.8. If $T_{1}, T_{2} \in \Omega_{V}$, define $T_{1} \leqq T_{2}$ to mean $\left(\exists U \in \Omega_{V}\right)\left(T_{1}+U=T_{2}\right)$.

THEOREM 2.9. If $T_{1}, T_{2} \in \Omega_{V}$, then $T_{1} \leqq T_{2}$ if and only if $\operatorname{dim}_{\alpha} T_{1} \leqq \operatorname{dim}_{\alpha} T_{2}$.

Proof. Suppose that $T_{1} \leqq T_{2}$. Then $T_{1}+U=T_{2}$ for some $U \in \Omega_{Y}$. Thus $\operatorname{dim}_{\alpha} T_{1}+\operatorname{dim}_{\alpha} U=\operatorname{dim}_{\alpha} T_{2}$, by Corollary 2.7 , and therefore $\operatorname{dim}_{\alpha} T_{1} \leqq \operatorname{dim}_{\alpha} T_{2}$.

Now suppose that $\operatorname{dim}_{\alpha} T_{1} \leqq \operatorname{dim}_{\alpha} T_{2}$. Then there is $X \in \Omega$ such that $\operatorname{dim}_{\alpha} T_{1}+X=\operatorname{dim}_{\alpha} T_{2}$. Now $X$ is equal to $\operatorname{dim}_{\alpha} U$ for some $U \in \Omega_{V}$, by the remark after Corollary 1.8, and so $\operatorname{dim}_{\alpha} T_{1}+\operatorname{dim}_{\alpha} U=\operatorname{dim}_{\alpha} T_{2}$. By Corollaries 2.7 and 1.8 , then, $T_{1}+U=T_{2}$, i.e. $T_{1} \leqq T_{2}$.

This theorem gives the reason why we had the restriction to $\Omega_{V}$ in Definition 2.8. If we had said $T_{1} \leqq T_{2}$ if there exists $U$ such that $T_{1}+U=T_{2}$ for any $T_{1}$ and $T_{2}$, then there would have been no guarantee that if $T_{1}$ and $T_{2}$ were members of $\Omega_{V}$ then the $U$ given by the definition would also be. We have not been able to discount the possibility that $\operatorname{dim}_{\alpha} T_{1}$ and $\operatorname{dim}_{\alpha}\left(T_{1}+U\right)$ be defined, but that $\operatorname{dim}_{\alpha} U$ be not defined.

Definition 2.10. If $V_{1}, V_{2} \in D$, then define $V_{1} \leqq V_{2}$ to mean there exists $V \in D$ such that $V_{1} \uparrow V=V_{2}$, and $V_{1}-\{0\}$ is recursively separable from $V-\{0\}$. (Here $\hat{f}$ is the standard algebraic linear sum operation.)

THEOREM 2.11. If $V_{1}, V_{2} \in D$, then $V_{1} \leqq V_{2}$ implies

(i) $\operatorname{dim}_{\alpha} V_{1} \leqq \operatorname{dim}_{\alpha} V_{2}$, and

(ii) $V$-RET $\left(V_{1}\right) \leqq V-R E T\left(V_{2}\right)$.

Finally in this section we prove:

THEOREM 2.12. If $T_{1}, T_{2} \in \Omega_{V}, T_{1} \leqq T_{2}$ and $T_{2} \leqq T_{1}$, then $T_{1}=T_{2}$.

Proof. By Theorem 2.9, we have $\operatorname{dim}_{\alpha} T_{1} \leqq \operatorname{dim}_{\alpha} T_{2}$ and $\operatorname{dim}_{\alpha} T_{2} \leqq \operatorname{dim}_{\alpha} T_{1}$. We can apply the Myhill-Cantor-Bernstein theorem (for R.E.T.s $X$ and $Y$, if $X \leqq Y$ and $Y \leqq X$, then $X=Y$ ), to get $\operatorname{dim}_{\alpha} T_{1}=\operatorname{dim}_{\alpha} T_{2}$. It follows that $T_{1}=T_{2}$, by Corollary 1.8 .

We shall see later how other theorems of this nature hold in $\Omega_{V}$ as a consequence of their holding in $\Omega$.

\section{Isolic spaces}

Dekker [2] has given many of the properties of isolic spaces. Here I want mainly to point out analogies with the properties of isolated sets and isols. First note that in general an isolic space (one with an isolated $\alpha$-basis) is not itself an isolated set, for if the field $F$ is infinite and if $v \in V-\{0\}$, then the set $\{x v: x \in F-\{0\}\}$ is an infinite r.e. subset of $V$.

Let $D_{\Lambda}=\left\{V \in D: \operatorname{dim}_{\alpha} V \in \Lambda\right\}$.

THEOREM 3.1. The following conditions are equivalent (assuming that $V \in D)$. 
(i) $V \in D_{\Lambda}$,

(ii) $V$ has no infinite dimensional r.e. subspace,

(iii) $W \subseteq V$ and $W \in D$ imply $W \in D_{\Lambda}$,

(iv) $W \subseteq V, W \in D, W^{\prime} \subseteq W$ and $W^{\prime} \simeq W$ together imply $W^{\prime}=W$,

(v) $W \subseteq V$ and $a \in U_{F}-\{0\}$ imply $W \neq W+V(\{a\})$, and

(vi) $W \subseteq V$ and $W+W_{1} \simeq W+W_{2}$, where $W, W_{1}, W_{2} \in D$, imply $W_{1}=W_{2}$.

Proof. (i) $\Rightarrow$ (ii) was proved by Dekker in [2].

(ii) $\Rightarrow$ (iii) is trivial.

(iii) $\Rightarrow$ (iv) is Lemma 3.2 below.

(iv) $\Rightarrow$ (i): Suppose that (iv) holds and that (i) does not. Then $V$ has an $\alpha$-basis $A$ which is not isolated. There is a partial recursive function $q$ such that $q: A \simeq A_{1}$, where $A_{1} \subseteq A$ properly. Obviously $A_{1}$ is an $\alpha$-basis for $V\left(A_{1}\right)$, and it can be shown easily that for such a $q$, the restriction of $q$ to $A$ extends to a partial recursive isomorphism $q^{\prime}: V(A) \simeq V\left(A_{1}\right)$. Now $V\left(A_{1}\right) \subseteq V$ properly, contradicting (iv). Thus (i) ...(iv) are equivalent.

(iii) $\Rightarrow(\mathrm{v})$ : By (iii) and Theorem 2.6, $\operatorname{dim}_{\alpha}(W+V(\{a\}))$ is an isol. By Lemma $3.2, W+V(\{a\}) \neq W$, since $W+V(\{a\})$ has a proper subspace which is $\alpha$-isomorphic to $W$.

(v) $\Rightarrow$ (i): By (v), $V \neq V+V(\{a\})$ for any $a \in U_{F}-\{0\}$. But if $\operatorname{dim}_{\alpha} V$ exists, then so does $\operatorname{dim}_{\alpha}(V+V(\{a\}))$, and

$$
\operatorname{dim}_{\alpha}(V+V(\{a\}))=\operatorname{dim}_{\alpha} V+1 .
$$

So we have $\operatorname{dim}_{\alpha} V \neq \operatorname{dim}_{\alpha} V+1$. Hence $\operatorname{dim}_{\alpha} V$ is an isol.

(vi) $\Rightarrow$ (v) is trivial.

(iii) $\Rightarrow$ (vi): Suppose that $W \subseteq V$ and $W+W_{1} \simeq W+W_{2}$, where $W, W_{1}$, $W_{2} \in D$. Then we have

$$
\operatorname{dim}_{\alpha} W+\operatorname{dim}_{\alpha} W_{1}=\operatorname{dim}_{\alpha} W+\operatorname{dim}_{\alpha} W_{2} .
$$

By (iii), however, $\operatorname{dim}_{\alpha} W$ is an isol, so we can deduce $\operatorname{dim}_{\alpha} W_{1}=\operatorname{dim}_{\alpha} W_{2}$. In turn this implies that $W_{1} \simeq W_{2}$.

The next lemma completes the proof of the theorem.

Lemma 3.2. If $V \in D_{\Lambda}, W \subseteq V$ and $p: V \simeq W$, then $W=V$.

Proof. Let $A=\left\{a_{i}: i<\omega\right\}$ be an $\alpha$-basis of $V$ and let $p: V \simeq W$, where $W \subseteq V$. Consider $a_{i} \in A$. Construct an r.e. subset $\mathrm{Cl}\left(a_{i}\right)$ of $A$ thus:

$\mathrm{Cl}\left(a_{i}\right)=\left\{a_{j} \in A\right.$ : there is a chain $a_{i_{0}}, \cdots, a_{i_{n}}$ of elements of $A$ such that $a_{i_{0}}=a, a_{i_{n}}=a_{j}$, and in the expression for $p\left(a_{i_{1}}\right)$ in terms of the basis $A, a_{i_{1+1}}$ appears with a nonzero coefficient, for $0 \leqq l<n\}$.

$A$ is isolated, so for each $i, \mathrm{Cl}\left(a_{i}\right)$ is finite. It is easily seen also that $p\left(V\left(\mathrm{Cl}\left(a_{i}\right)\right)\right)=V\left(\mathrm{Cl}\left(a_{i}\right)\right)$ for each $i$, since $V\left(\mathrm{Cl}\left(a_{i}\right)\right)$ is finite dimensional and $p$ is one-one. Now $a_{i} \in V\left(\mathrm{Cl}\left(a_{i}\right)\right)$, so $a_{i} \in \rho p$ for each $i$, and further, $A$ is contained in the image of $V$ under $p$. It follows that $p(V)=V$, and so $W=V$. 
LEMmA 3.3. If $V_{1}, V_{2} \in D_{\Lambda}$, then $V_{1}+V_{2} \in D_{\Lambda}$.

LEMmA 3.4. (i) If $V_{1} \in D, V_{2} \in D_{\Lambda}$ and $V_{1} \leqq V_{2}$, then $V_{1} \in D_{\Lambda}$.

(ii) If $T_{1} \in \Omega_{\Lambda}, T_{2} \in \Lambda_{V}$ and $T_{1} \leqq T_{2}$, then $T_{1} \in \Lambda_{V}$.

\section{Relations}

Here we deal with V-R.E.T.s and relations among them. Our aim is the following:

THEOREM 4.1. Suppose that $\phi$ is a (finite) formula involving + , $\leqq$, varibales, logical connectives and first order quantifiers. Then $\phi$ holds universally in $\Omega_{V}$ (respectively in $\Lambda_{V}$ ) if and only if $\phi$ holds universally in $\Omega$ (respectively in $\Lambda)$.

The remainder of the paper is devoted to proving this, but first some preliminaries.

Notation. If $R \subseteq \mathbf{X}^{k} \Omega$, i.e. if $R$ is a relation among R.E.T.s, let

If

$$
R_{V}=\left\{\left(T_{1}, \cdots, T_{k}\right) \in \mathbf{X}^{k} \Omega_{V}:\left(\operatorname{dim}_{\alpha} T_{1}, \cdots, \operatorname{dim}_{\alpha} T_{k}\right) \in R\right\} .
$$

$$
R=\left\{\left(X_{1}, \cdots, X_{k}\right) \in \mathbf{X}^{k} \Omega: \phi\left(X_{1}, \cdots, X_{k}\right)\right\},
$$

where $\phi$ is a formula as described in Theorem 4.1, let

$$
R^{\prime}=\left\{\left(T_{1}, \cdots, T_{k}\right) \in \mathbf{X}^{k} \Omega_{V}: \phi\left(T_{1}, \cdots, T_{k}\right)\right\} .
$$

THEOREM 4.2. Suppose that $R \subseteq \mathbf{X}^{k} \Omega$. Then

(i) $R=\mathbf{X}^{k} \Omega$ if and only if $R_{Y}=\mathbf{X}^{k} \Omega_{V}$, and

(ii) $R=\mathbf{X}^{k} \Lambda$ if and only if $R_{V}=\mathbf{X}^{k} \Lambda_{V}$.

Proof. Suppose that $R=\mathbf{X}^{k} \Omega$. If $\left(T_{1}, \cdots, T_{k}\right) \in \mathbf{X}^{k} \Omega_{V}$ then $\left(\operatorname{dim}_{\alpha} T_{1}, \cdots, \operatorname{dim}_{\alpha} T_{k}\right) \in \mathbf{X}^{k} \Omega=R$. Hence $\left(T_{1}, \cdots, T_{k}\right) \in R_{V}$.

Now suppose that $R_{V}=\mathbf{X}^{k} \Omega_{V}$. If $\left(X_{1}, \cdots, X_{k}\right) \in \mathbf{X}^{k} \Omega$ then there exist $\left(T_{1}, \cdots, T_{k}\right) \in \mathbf{X}^{k} \Omega_{Y}$ such that for $1 \leqq i \leqq k, \operatorname{dim}_{\alpha} T_{i}=X_{i}$. By the definition of $R_{V}$, then, $\left(\operatorname{dim}_{\alpha} T_{1}, \cdots, \operatorname{dim}_{\alpha} T_{k}\right) \in R$, i.e. $\left(X_{1}, \cdots, X_{k}\right) \in R$. Hence $R=X^{k} \Omega$.

Part (ii) is proved in the same way.

THEOREM 4.3. If $S \subseteq \mathbf{X}^{k} \Omega_{V}$, then $S=R_{V}$ for some $R \subseteq \mathbf{X}^{k} \Omega$.

Proof. Let $S_{D}=\left\{\left(X_{1}, \cdots, X_{k}\right) \in \mathbf{X}^{k} \Omega\right.$ : there exists $\left(T_{1}, \cdots, T_{k}\right) \in \mathbf{X}^{k} \Omega_{V}$ such that for $1 \leqq i \leqq k, \operatorname{dim}_{\alpha} T_{i}=X_{i}$ and $\left.\left(T_{1}, \cdots, T_{k}\right) \in S\right\}$.

It is easy to verify that $\left(S_{D}\right)_{V}=S$.

LEMMA 4.4. Suppose that

$$
R=\left\{\left(X_{1}, \cdots, X_{m+n}\right) \in \mathbf{X}^{m+n} \Omega: X_{1}+\cdots+X_{m} \leqq X_{m+1}+\cdots+X_{m+n}\right\},
$$

and that $R^{\prime}$ is as described above. 
Then $R^{\prime}=R_{V}$.

Proof. Using Corollary 2.7 and Theorem 2.9,

$$
\begin{aligned}
R_{V} & =\left\{\left(T_{1}, \cdots, T_{m+n}\right):\left(\operatorname{dim}_{\alpha} T_{1}, \cdots, \operatorname{dim}_{\alpha} T_{m+n}\right) \in R\right\} \\
& =\left\{\left(T_{1}, \cdots, T_{m+n}\right): \operatorname{dim}_{\alpha} T_{1}+\cdots+\operatorname{dim}_{\alpha} T_{m} \leqq \operatorname{dim}_{\alpha} T_{m+1}+\cdots+\operatorname{dim} T_{m+n}\right\} \\
& =\left\{\left(T_{1}, \cdots, T_{m+n}\right): \operatorname{dim}_{\alpha}\left(T_{1}+\cdots+T_{m}\right) \leqq \operatorname{dim}_{\alpha}\left(T_{m+1}+\cdots+T_{m+n}\right)\right\} \\
& =\left\{\left(T_{1}, \cdots, T_{m+n}\right): T_{1}+\cdots+T_{m} \leqq T_{m+1}+\cdots+T_{m+n}\right\} \\
& =R^{\prime} .
\end{aligned}
$$

This is the base step of our inductive proof of Theorem 4.1, and now follow the induction steps.

Lemma 4.5. Suppose that $\phi_{1}$ and $\phi_{2}$ are formulas as described in Theorem 4.1, and that

$$
\begin{aligned}
& R_{1}=\left\{X \in \mathbf{X}^{k} \Omega: \phi_{1}(X)\right\}, \\
& R_{2}=\left\{X \in \mathbf{X}^{k} \Omega: \phi_{2}(X)\right\} .
\end{aligned}
$$

Then if $R_{1}^{\prime}=\left(R_{1}\right)_{V}$ and $R_{2}^{\prime}=\left(R_{2}\right)_{V}$, we have

(i) $\left(\mathbf{X}^{k} \Omega-R_{1}\right)^{\prime}=\left(\mathbf{X}^{k} \Omega-R_{1}\right)_{V}$,

(ii) $\left(R_{1} \cup R_{2}\right)^{\prime}=\left(R_{1} \cup R_{2}\right)_{V}$, and

(iii) $\left(R_{1} \cap R_{2}\right)^{\prime}=\left(R_{1} \cap R_{2}\right)_{V}$.

Proof. It is easily shown that

$$
\begin{aligned}
\left(\mathbf{X}^{k} \Omega-R_{1}\right)_{V} & =\mathbf{X}^{k} \Omega_{V}-\left(R_{1}\right)_{V} \\
& =\mathbf{X}^{k} \Omega_{V}-R_{1}^{\prime} \text { by hypothesis, } \\
& =\left(\mathbf{X}^{k} \Omega-R_{1}\right)^{\prime} .
\end{aligned}
$$

The proofs of (ii) and (iii) are similar.

LeMMA 4.6 (i) Let $\phi_{1}, R_{1}$ be as above, and let $\phi$ be the formula $\left(\forall x_{1}\right) \phi_{1}\left(x_{1}\right)$, where $x_{1}$ is a variable which does not occur bound in $\phi_{1}$. Now let

$$
R=\left\{\left(X_{2}, \cdots, X_{k}\right) \in \mathbf{X}^{k-1} \Omega:\left(\forall X_{1} \in \Omega\right) \phi_{1}\left(X_{1}, \cdots, X_{k}\right)\right\} .
$$

If $R_{1}^{\prime}=\left(R_{1}\right)_{V}$, then $R^{\prime}=R_{V}$.

(ii) As (i), but with an existential quantifier.

ProOF.

$$
\begin{aligned}
R_{V} & =\left\{\left(T_{2}, \cdots, T_{k}\right):\left(\operatorname{dim}_{\alpha} T_{2}, \cdots, \operatorname{dim}_{\alpha} T_{k}\right) \in R\right\} \\
& =\left\{\left(T_{2}, \cdots, T_{k}\right):\left(\forall X_{1} \in \Omega\right) \phi_{1}\left(X_{1}, \operatorname{dim}_{\alpha} T_{2}, \cdots, \operatorname{dim}_{\alpha} T_{k}\right)\right\} \\
& =\left\{\left(T_{2}, \cdots, T_{k}\right):\left(\forall T_{1} \in \Omega_{V}\right) \phi_{1}\left(\operatorname{dim}_{\alpha} T_{1}, \cdots, \operatorname{dim}_{\alpha} T_{k}\right)\right\},
\end{aligned}
$$




$$
\begin{gathered}
\text { by the final remark of Section 1, } \\
=\left\{\left(T_{2}, \cdots, T_{k}\right):\left(\forall T_{1} \in \Omega_{V}\right)\left(\left(\operatorname{dim}_{\alpha} T_{1}, \cdots, \operatorname{dim}_{\alpha} T_{k}\right) \in R_{1}\right)\right\} \\
=\left\{\left(T_{2}, \cdots, T_{k}\right):\left(\forall T_{1} \in \Omega_{V}\right)\left(\left(T_{1}, \cdots, T_{k}\right) \in\left(R_{1}\right)_{V}\right)\right\} \\
=\left\{\left(T_{2}, \cdots, T_{k}\right):\left(\forall T_{1} \in \Omega_{V}\right)\left(\left(T_{1}, \cdots, T_{k}\right) \in R_{1}^{\prime}\right)\right\}, \\
\quad \text { by our hypothesis, } \\
=\left\{\left(T_{2}, \cdots, T_{k}\right):\left(\forall T_{1} \in \Omega_{V}\right) \phi_{1}\left(T_{1}, \cdots, T_{k}\right)\right\} \\
=R^{\prime} .
\end{gathered}
$$

Part (ii) follows from (i) and the previous lemma.

We combine the last three lemmas now into:

LEMma 4.7. If $\phi$ is as described in Theorem 4.1, and $R=\left\{X \in \mathbf{X}^{k} \Omega: \phi(X)\right\}$, then $R^{\prime}=R_{V}$.

Now we invoke Theorem 4.2 to obtain

(i) $R=X^{k} \Omega$ if and only if $R^{\prime}=X^{k} \Omega_{Y}$, and

(ii) $R=\mathbf{X}^{k} \Lambda$ if and only if $R^{\prime}=\mathbf{X}^{k} \Lambda_{V}$.

Theorem 4.1 is therefore proved.

Notes: (i) If $\phi$ is a closed formula, then to apply the above we may just introduce a redundant free variable.

(ii) Because of Theorem 2.12, it was not necessary to include " $=$ " as one of the symbols from which the admissible formulas $\phi$ were composed. However, we could just as well have included " $=$ " in the statement of Theorem 4.1. Theorem 2.12 would then have been a corollary.

Finally, Theorem 4.1, along with results of Nerode [7] and Manaster and Nerode [6], enable us to state the following.

THEOREM 4.8. (i) Universal sentences about the structure $\left(\Omega_{V},+, \leqq\right)$ are decidable.

(ii) The first order theory of $\left(\Omega_{V},+, \leqq\right)$ is not decidable.

Proof. Theorem 4.1 says that the first order theories of $\left(\Omega_{V},+, \leqq\right)$ and $(\Omega,+, \leqq)$ are the same. In $[7]$ it is proved that universal sentences about $(\Omega,+, \leqq)$ are decidable, so (i) follows. In [6] it is shown that the first order theory of $(\Omega,+)$ is not decidable, so (ii) follows.

\section{Conclusion}

The reason why vector spaces inherit properties of the kind discussed from their $\alpha$-bases is that vector spaces are "freely generated" from sets with no structure. Linear independence is really just an absence of structure. This suggests 
that results similar to the above may be obtained for free algebraic systems more general than vector spaces. That is indeed the case, and some research in this direction is described in [5].

\section{References}

[1] J. C. E. Dekker: 'On certain vector spaces of isolic dimension, Part 1', Abstract. J. Symb. Logic 33 (1968), 642.

[2] J. C. E. Dekker, 'Countable vector spaces with recursive operations, Part 1', J. Symb. Logic 34 (1969), 363-387.

[3] J. C. E. Dekker, and J. Myhill, 'Recursive equivalence types', Univ. Calif. Publs. in Math. (New Series) 3, No. 3 (1960), 67-214.

[4] A. G. Hamilton, 'Bases and $\alpha$-dimensions of countable vector spaces with recursive operations', J. Symb. Logic 35 (1970), 85-96.

[5] A. G. Hamilton, Mathematical structures and recursive equivalence. D. Phil. thesis, (Oxford (1969)).

[6] A. B. Manaster, and A. Nerode, 'A universal embedding property of the RETs', J. Symb. Logic 35 (1970), 51-59.

[7] A. Nerode, 'Additive relations among recursive equivalence types', Math. Annalen 159 (1965), 329-343.

Department of Mathematics

University of Stirling

Scotland 